\title{
Reflets
}

Revue ontaroise d'intervention sociale et communautaire

\section{Bilan des stratégies pour combattre la pauvreté chez les enfants et les adolescents à Sudbury}

\section{Marge Reitsma-Street, Adje van de Sande et François Boudreau}

Volume 3, numéro 1, printemps 1997

Enfance et familles en contexte d'appauvrissement

URI : https://id.erudit.org/iderudit/026155ar

DOI : https://doi.org/10.7202/026155ar

Aller au sommaire du numéro

Éditeur(s)

Reflets : Revue ontaroise d'intervention sociale et communautaire

ISSN

1203-4576 (imprimé)

1712-8498 (numérique)

Découvrir la revue

Citer cet article

Reitsma-Street, M., van de Sande, A. \& Boudreau, F. (1997). Bilan des stratégies pour combattre la pauvreté chez les enfants et les adolescents à Sudbury.

Reflets, 3(1), 120-135. https://doi.org/10.7202/026155ar

Tous droits réservés @ $\odot$ Reflets : Revue ontaroise d'intervention sociale et communautaire, 1997
Ce document est protégé par la loi sur le droit d'auteur. L’utilisation des services d’Érudit (y compris la reproduction) est assujettie à sa politique d'utilisation que vous pouvez consulter en ligne.

https://apropos.erudit.org/fr/usagers/politique-dutilisation/ 


\title{
Bilan des stratégies pour combattre la pauvreté chez les enfants et les adolescents à Sudbury
}

\author{
Marge Reitsma-Street, Adje van de Sande et François Boudreau ${ }^{1}$ \\ Université Laurentienne
}

\section{Introduction}

Le 24 novembre 1989, la Chambre des communes a adopté à l'unanimité une motion présentée par M.Edward Broadbent, alors chef du Nouveau Parti Démocratique, qui se lisait ainsi : "Que la Chambre témoigne de sa préoccupation pour le million et plus d'enfants Canadiens qui vivent sous le seuil de pauvreté et s'emploie à réaliser l'objectif d'éliminer la pauvreté chez les enfants au Canada d'ici l'an 2000» (Rapport du Comité permanent de la santé et du bien-être social, des affaires sociales, du troisième âge et de la condition féminine, 1993). Au Canada, un enfant sur cinq est élevé dans la pauvreté. Ces enfants représentent le plus grand groupe de personnes pauvres.

Le portrait global de la pauvreté chez les jeunes au Canada est bien connu, mais qu'en est-il de la situation des jeunes dans la région de Sudbury? La municipalité régionale de Sudbury se trouve dans le Nord-Est de l'Ontario, à $400 \mathrm{~km}$ au nord de Toronto. Elle comprend la ville de Sudbury et six petites municipalités adjacentes ${ }^{2}$. Près du tiers de sa population a le français pour langue maternelle, comparativement au taux provincial de 5,4\%. En 1991, $28 \%$ des 162000 résidents étaient âgés de 18 ans ou moins. L'industrie minière, pierre angulaire de l'économie sudburoise depuis la fin 
du XIX ${ }^{e}$ siècle, demeure prépondérante dans la région, même si les années quatre-vingt ont vu le développement d'emplois dans la fonction publique et dans le secteur des services, contribuant à la diversification de l'économie régionale.

Ce trop bref retour sur la situation historique et sociale de la région de Sudbury donne tout de même une idée de ses principales caractéristiques. Dans cet article, nous dresserons un bilan des étapes et des stratégies déployées par un collectif ${ }^{3}$ de personnes et d'organismes de la région de Sudbury pour combattre la pauvreté entre 1989 et 1996 . Nous résumerons également les résultats de deux études sur l'étendue, la profondeur et l'impact de la pauvreté sur la vie des enfants et des adolescents de Sudbury.

\section{Première étape : un colloque}

Au début de l'année 1988, le Comité d'examen de l'aide sociale en Ontario publie un rapport intitulé Transition. Ce rapport indique qu'un large pourcentage des bénéficiaires de l'assistance sociale est constitué d'enfants.

La méthode utilisée pour chercher les données sur le taux de personnes bénéficiant de l'assistance sociale (en Ontario) a démontré un fait révélateur: $37 \%$ de tous les bénéficiaires - 205000 personnes - sont des enfants de moins de dix-huit ans. (Rapport du Comité d'examen de l'aide sociale 1988: 14)

De plus, ce même rapport démontre l'insuffisance des prestations de l'assistance sociale pour aider les prestataires à sortir de la pauvreté; ces prestations ne sont même pas suffisantes pour permettre aux gens de couvrir adéquatement leurs dépenses de base (logement, nourriture, vêtements).

Le Rapport du Comité d'examen de l'aide sociale (1988) a incité certains membres de l'École de service social de l'Université Laurentienne à solliciter une aide financière auprès du ministère des Services sociaux et communautaires, en 1989, pour organiser un colloque sur la pauvreté des enfants dans la région de Sudbury. 
Des bénéficiaires de l'aide sociale, des intervenants et intervenantes, des étudiants et étudiantes, des professeurs et professeures de l'École de service social, ainsi qu'une représentante de Campagne 2000 ont participé à ce colloque. Campagne 2000 est l'organisme qui travaille à l'élimination de la pauvreté chez les enfants au Canada. Les allocutions ont porté sur l'étendue de la pauvreté au Canada, sur les effets sociaux et individuels de la pauvreté des enfants et sur l'urgence de s'occuper de ce problème social. À la fin du colloque, les participants ont constaté le manque d'information au sujet de l'étendue de la pauvreté chez les enfants de la région de Sudbury. Les organisateurs ont alors développé un projet de recherche visant à rassembler les faits devant donner une image claire de la situation de la pauvreté chez les enfants dans la région de Sudbury.

\section{Deuxième étape : le rapport de 1992}

Le rapport sur la pauvreté des enfants de la région de Sudbury, principal objectif issu de la conférence de 1989, fut publié en 1992 (Carrière, Hein, Reitsma-Street, van de Sande 1992). Il comporte deux objectifs généraux: d'abord celui de dresser un portrait détaillé de la situation des enfants vulnérables à la pauvreté sociale et économique dans la région de Sudbury et ensuite celui de promouvoir un débat sur les solutions régionales au problème de la pauvreté. Il s'agit de faire reconnaître à la population de la région de Sudbury l'existence, dans leurs villes, de milliers d'enfants pauvres.

En se basant sur les données du recensement de 1986, le rapport de 1992 souligne que 19,4 pourcent des enfants et des adolescents de la région de Sudbury vivent dans des foyers dont le revenu est inférieur au seuil de pauvreté établi par Statistique Canada. En 1990, plus de 7000 enfants et adolescents de la région de Sudbury dépendent de l'aide sociale pour leurs besoins essentiels en matière de logement, de nourriture et de vêtements. Aussi, le rapport indique que ces prestations ne suffisent pas à combler les besoins essentiel, puisque au moins 5000 de ces jeunes avaient dû recourir aux banques alimentaires au moins une fois en 1990 . 


\section{Troisième étape : travail auprès des médias}

Afin de conscientiser la population de Sudbury aux réalités de la pauvreté, les médias ont été invités à participer à une campagne publicitaire. Tous les postes de radio et de télévision de la région, ainsi que tous les journaux de la ville ont présenté des reportages sur les résultats de la recherche.

A titre d'exemple, le Sudbury Star du 21 mai 1992 titre à la une: Poverty Grips Thousands of Local Kids. L'article poursuit en indiquant: Study finds the incidence of poverty in Sudbury and the surrounding area is higher than the provincial and national average (Pender 1992). Un autre exemple, tiré cette fois du journal Le Voyageur du 27 mai 1992, indique que :

En 1981 et 1986, 5565 des 28720 (soit 19,4\%) enfants de la ville de Sudbury vivaient dans des foyers dont le revenu était inférieur au senil de pauvreté établi par Statistique Canada. Pour régler le problème, la

«Peu de temps après la publication des résultats du rapport, le Conseil de planification sociale de Sudbury a effectué un sondage pour obtenir l'opinion de la population de Sudbury au sujet des problèmes sociaux les plus préoccupants. $\mathrm{La}$ pauvreté chez les enfants et les adolescents compte parmi les plus importants.» population entière doit faire preuve de volonté et d'engagement. Étant donné que la Commission de développement économique de Sudbury travaille présentement à l'élaboration de son plan de développement des dix prochaines années, on espère que l'on pourra arriver à une vision qui tiendra compte $d u$ problème de la pauvreté qui affecte la vie de 9420 enfants de la région de Sudbury (de la Riva 1992).

La publicité obtenue suite à la conférence de presse a l'effet désiré. Peu de temps après la publication des résultats du rapport, le Conseil de planification sociale de Sudbury a effectué un sondage pour obtenir l'opinion de la population de Sudbury au sujet des problèmes sociaux les plus préoccupants. La pauvreté chez les enfants et les adolescents compte parmi les plus importants. De plus, en 1993, le Conseil régional de la municipalité de Sudbury adopte une perspective communautaire qui souligne l'importance des conditions de vie des enfants et des jeunes. 


\section{Quatrième étape : développement d'un «collectif d'intervention»}

La question de la pauvreté chez les enfants et les adolescents est rapidement devenue une priorité pour plusieurs groupes et organismes de la région. Suite à la publication du premier rapport sur la pauvreté, plusieurs se montrent intéressés à participer à des activités pour combattre la pauvreté. Un collectif impressionnant d'organismes sociaux et communautaires se forme. L'École de service social de l'Université Laurentienne, le Centre de femmes de Sudbury, le Conseil des écoles publiques de Sudbury, l'Unité de santé du district de Sudbury, la banque alimentaire Inner City Home, le Child Poverty Action Group, les Services de santé mentale des enfants, le Département de services sociaux de Sudbury, le ministère des Services sociaux et communautaires, le Groupe consultatif et de recherche pour le service social, le Centre de recherche en développement humain et le Conseil de planification sociale de Sudbury en font partie.

Quelques-uns de ces organismes participent à plusieurs projets destinés à combattre la pauvreté chez les enfants (Plouffe 1993; Pilon 1993). Soulignons d'abord le projet de recherche mené sous les auspices de l'Unité de santé du district de Sudbury, à l'été 1993, pour étudier la relation entre la pauvreté et la santé chez les enfants. Cette recherche débouche sur le développement du programme Nutriaction, dont l'objectif est de promouvoir une nutrition saine et équilibrée pour les jeunes écoliers. Un étudiant de l'École de service social travaille avec le Conseil des écoles publiques de Sudbury pour étudier l'incidence des frais afférents sur les familles dont les enfants fréquentent les écoles publiques. Une autre étudiante fait une étude sur le nombre d'enfants qui sont des bénéficiaires des banques alimentaires à Sudbury. Soulignons également le colloque de février 1994 intitulé Revealing Strengths Youth Conference et organisé par des jeunes en collaboration avec le Conseil de planification sociale de Sudbury, ayant permis aux 75 jeunes participantes et participants de parler des problèmes qu'ils vivaient, y compris ceux directement liés à la pauvreté. 


\section{Cinquième étape : rapport de 1994}

L'équipe de recherche de l'École de service social décide de s'engager dans une deuxième étude, en utilisant cette fois les données du recensement de 1991, et en développant une section spécifique sur les stratégies pour combattre la pauvreté. Parmi les groupes impliqués dans le développement de ce deuxième rapport on retrouve entre autres, le Groupe consultatif et de recherche pour le service social, l'Unité de santé du district de Sudbury, le Centre de recherche en développement humain, et l'École de service social (Reitsma-Street 1994a).

En utilisant des tabulations spéciales des données du recensement de Statistique Canada, cette recherche démontre qu'en 1991, $21,4 \%$ des adolescents et des enfants sudburois de moins de dixhuit ans vivent dans des familles dont le revenu se situe en-dessous du seuil de pauvreté établi par Statistique Canada. Ce seuil est établi à 21400 \$ pour une famille de trois personnes dans une ville de grandeur moyenne (Rapport du Conseil national du bienêtre social 1991). Cette étude permet de constater qu'un enfant pauvre sur trois vit dans une situation de pauvreté extrême, sa famille bénéficiant d'un revenu annuel de moins de $10000 \$$ (Hein 1994).

Tableau 1

Nombre et pourcentage d'enfants pauvres par région (moins de 18 ans)

\begin{tabular}{lcc}
\hline Région & Nombre d'enfants & Pourcentage \\
\hline Ville de Sudbury & 3730 & $21,4 \%$ \\
Région de Sudbury & 5605 & $16,4 \%$ \\
Ontario & 346000 & $14,7 \%$ \\
Canada & 1105000 & $16,9 \%$ \\
\hline
\end{tabular}

Depuis 1980, le taux de pauvreté des familles de la région de Sudbury demeure considérablement plus élevé que les taux ontarien et canadien. Le tableau 1 indique un taux de pauvreté de $21,4 \%$ pour la ville de Sudbury en 1991, alors qu'il se situe à $14,7 \%$ et à $16,9 \%$ respectivement pour l'Ontario et pour le 
Canada. Au cours de la dernière décennie, le taux de pauvreté de la ville de Sudbury a connu un mouvement parallèle à celui du chômage, augmentant et diminuant en même temps que ce dernier, tout en demeurant toujours plus élevé que le taux de pauvreté provincial.

La région de Sudbury est composée d'une population de cultures variées. Elle peut être qualifiée de région multiculturelle. En plus des anglophones et des francophones qui forment son noyau, la région comprend un fort contingent d'Amérindiens. Le tiers de la population de la région indique le français comme langue maternelle. Dans deux des cinq municipalités de la région, la population est à majorité francophone. À première vue, les taux de pauvreté entre les groupes culturels semblent comporter peu de différences. Cependant, les renseignements incomplets n'ont pas permis d'obtenir précisement le taux de pauvreté de la population amérindienne, ni de comparer ce taux avec ceux des groupes culturellement dominants.

Tableau 2

\begin{tabular}{lcc}
\hline & francophones & anglophones \\
\hline $\begin{array}{l}\text { Les enfants de la ville de Sudbury } \\
\text { Les enfants de la région de Sudbury }\end{array}$ & $16,5 \%$ & $15,6 \%$ \\
$\begin{array}{l}\text { Les enfants des familles monoparentales } \\
\text { de la ville de Sudbury }\end{array}$ & $12,6 \%$ \\
$\begin{array}{l}\text { Les enfants des familles monoparentales } \\
\text { de la région de Sudbury }\end{array}$ & $51,3 \%$ \\
\hline
\end{tabular}

Le tableau 2 présente le taux de pauvreté infantile de la population francophone. Celui-ci est un peu plus élevé que le taux de pauvreté infantile de la population anglophone de Sudbury. Les différents taux de pauvreté des petites villes de la région se lisent comme suit: 14,5\% des enfants et adolescents de Rayside-Balfour, municipalité francophone à $62 \%$ et $8,9 \%$ des enfants et adolescents de Valley East, municipalité francophone à $49 \%$, sont pauvres.

Contrairement au cliché qui dit que les familles pauvres ont beaucoup d'enfants, les familles pauvres de la région de Sudbury 
ont peu d'enfants; $80 \%$ de ces familles comptent deux enfants ou moins. La même constatation s'applique à tous les types de familles. Seulement $22 \%$ des familles monoparentales ont plus de deux enfants. Il n'en demeure pas moins que les familles monoparentales de la région, qui constituent $14 \%$ de toutes les familles, sont plus vulnérables à la pauvreté. Deux tiers des enfants et des adolescents pauvres vivent dans une famille monoparentale. Ce chiffre a doublé par rapport à 1981.

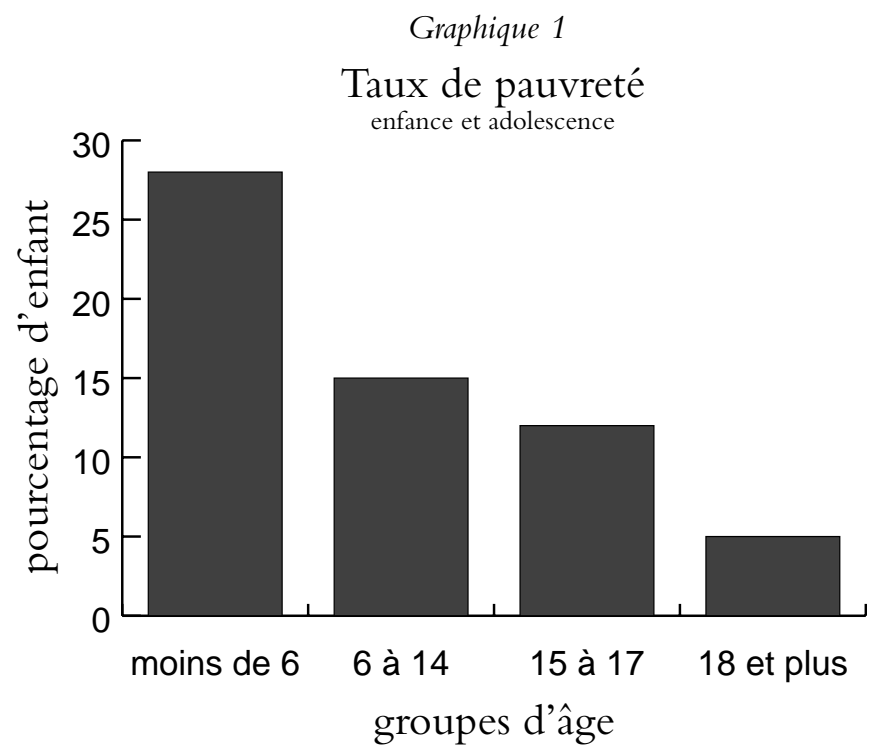

Le graphique 1 montre clairement que plus les enfants sont jeunes, plus ils sont susceptibles de vivre dans des foyers pauvres. Plus d'un enfant sur quatre $(27,3 \%)$ âgés de moins de six ans était pauvre en 1990 dans la Ville de Sudbury. Une des raisons qui explique cette situation est que les jeunes parents de ces enfants occupent plus souvent des emplois à temps partiel ou ont des salaires inférieurs à ceux qui sont plus âgés. Une autre raison expliquant cette vulnérabilité à la pauvreté, surtout chez les jeunes enfants et chez ceux qui se trouvent dans des familles dont le chef est une femme, est le fait que le revenu des femmes sudburoises en 1990 est inférieur, souvent de beaucoup, à celui des hommes. 
Il se trouve même en-dessous de la moyenne provinciale du salaire des femmes.

Au mois de décembre 1991, alors que la récession a frappé Sudbury et que les modifications au régime d'assurance chômage ont limité l'accès, $19 \%$ des adolescents et des enfants de la région de Sudbury vivent dans des familles qui dépendent de l'aide sociale (Carrière \& van de Sande 1994). Quarante-trois pour cent des enfants de foyers recevant de l'aide sociale ont moins de six ans.

En décembre 1991, près de $50 \%$ des adultes qui fréquentent les comptoirs alimentaires ont des enfants. Entre 1987 et 1991, un enfant sur deux qui bénéficie de ces services à Sudbury a moins de six ans. Comme l'indique cette remarque d'un enfant, ce ne sont pas seulement les adultes, avec ou sans enfants, qui ont recours aux comptoirs alimentaires : "Quand le directeur de l'école a demandé à un élève pourquoi il était en retard, l'élève a répondu qu'il était obligé d'aller à une banque alimentaire pour chercher de la nourriture pour lui et sa soeur» (Reitsma-Street 1994b:36).

Selon les données recueillies à l'accueil de deux organismes importants dans le domaine du logement-secours à Sudbury, en 1990, plus de 1300 enfants et adolescents sudburois vivent dans

«De plus, du nombre total des enfants admis avec leur mère à un logement-secours sans passer par le service de police, $74 \%$ ont $d u$ quitter le logement secours dans la semaine suivant leur arrivée. Cette situation est le résultat $d u$ financement provincial insuffiant de ces centres de logementsecours.» des logements peu adéquats. De plus, du nombre total des enfants admis avec leur mère à un logement-secours sans passer par le service de police, $74 \%$ ont du quitter le logement secours dans la semaine suivant leur arrivée. Cette situation est le résultat du financement provincial insuffiant de ces centres de logement-secours.

Un sondage sur la santé effectué en Ontario en 1990 souligne que les adolescents des familles à faible revenu qui vivent dans le Nord-Est ont un plus faible niveau de satisfaction concernant leur état de santé que les adolescents des familles dont le revenu était plus élevé (Sahai 1994). Il n'est pas surprenant de constater que ces jeunes vont également plus souvent chez le médecin. Lorsqu'il faut payer, comme c'est le cas chez le dentiste, les adolescents des familles démunies rapporte un moins grand nombre de visites que les adolescents des familles à revenu plus élevé.

Parmi les thèmes qui ressortent des discussions avec les parents prestataires de l'aide sociale à Sudbury, en 1993, le plus frappant 
concerne les idées préconçues liées à l'aide sociale. Les enfants en particulier subissent les préjugés des autres enfants du fait de leur condition d'assistés sociaux. Un parent témoigne : «Mon fils m’a même dit que les enfants à l'école disaient qu'il portait des microbes à cause du fait qu'il était pauvre. Il m'a demandé s'il avait des microbes. J'étais obligé de dire que ces enfants étaient cruels.» (Hein et al. 1994: 57). Un autre thème de très grande importance soulevé lors de ces mêmes discussions se rapporte au développement et au maintien de l'estime de soi. Les enfants des familles qui dépendent de l'aide sociale doivent constamment lutter contre les stéréotypes, se défendre de n'avoir pas de vêtements à la mode, se justifier de demeurer dans des logements moins beaux, etc. Ces luttes constantes minent leur sentiment d'appartenance au groupe et affaiblissent leur confiance en eux-mêmes.

Les parents avec lesquels nous avons parlé ont aussi exprimé d'importantes préoccupations face aux difficultés de répondre aux besoins essentiels quotidiens de leurs enfants, particulièrement aux niveaux des loisirs, de la nourriture, du logement, du gardiennage et des dépenses scolaires.

\section{Sixième étape : stratégies proposées et stratégies utilisées}

Le collectif de lutte contre la pauvreté a proposé quelques stratégies que les organismes pourraient utiliser dans leurs milieux respectifs

"Une étape stratégique importante consiste à augmenter le niveau de sensibilisation $d u$ public face à la pauvreté, dans le but d'élargir le débat autour des enjeux et de développer des pistes de solutions efficaces et durables à la pauvreté.»
(Reitsma-Street 1994c: 74). Certaines de ces stratégies ont déjà été mises en pratique. Une étape stratégique importante consiste à augmenter le niveau de sensibilisation du public face à la pauvreté, dans le but d'élargir le débat autour des enjeux et de développer des pistes de solutions efficaces et durables à la pauvreté. Dans cette optique, l'École de service social a organisé une conférence de presse pour les médias du Nord-Est ontarien en 1993. Une autre conférence de presse, en 1994, a coïncidé avec l'anniversaire de la proposition à la Chambre des Communes visant à éliminer la pauvreté des enfants avant l'an 2000. De nombreux articles ont été publiés dans les journaux francophones et anglophones (Girouard 1994; Pender 1994; Plouffe 1994). Des représentants de chacun des 29 organismes membres du collectif ont distribué 
le rapport de 1994 et ont fait des présentations dans leur organisme respectif. Les auteurs du rapport, invités à la radio et dans les assemblées annuelles des divers organismes, font valoir l'urgence d'un examen de la situation d'ensemble de la pauvreté; la compilation des coûts de l'aide sociale doit tenir compte à la fois des coupures gouvernementales et du taux de chômage dans un contexte large où tous les éléments sont interreliés.

La deuxième stratégie vise à ce que chaque groupe, association, organisme et membre du collectif fasse de la question de la pauvreté des enfants une priorité dans son travail. Les discussions autour de cette stratégie soulèvent l'importance de la participation active des adolescents et des parents dans l'élaboration de plans d'action concrets et simples, réalisables rapidement et à faible coût. Chaque organisme doit dresser un plan d'action précisant ce qui peut se faire dans son domaine de compétence respectif. De plus, chaque organisme s'engage à examiner les politiques et les pratiques existantes dans son milieu qui, d'une façon insidieuse ou autre, sont suceptibles d'exacerber les facteurs contribuant à l'appauvrissement des familles à faible revenu. Cet excercice vise, bien entendu, l'élimination de ces pratiques.

Par exemple, après les présentations et les discussions avec les directeurs et les surintendants des écoles de la région au sujet des rapports de 1992 et de 1994 sur la pauvreté des adolescents, le Conseil des écoles publiques de Sudbury décide d'étudier, à l'automne 1994, la question des demandes financières faites auprès des étudiants et de leurs parents. Le Conseil scolaire veut ainsi évaluer l'impact des dépenses scolaires non prévues ainsi que des demandes de prélèvement de fonds sur les adolescents pauvres et leur famille (Brown 1994). Ce même Conseil a mis sur pied un comité d'étude dont la moitié des membres sont des parents et des représentants d'agences.Après la tenue d'audiences publiques, de nouvelles politiques sont annoncées, en juin 1995, visant à surveiller de plus près les modalités et les montants permis lors de prélèvements de fonds ( Sudbury Board of Education 1995). Ces politiques permettent d'aviser les parents avant que des demandes d'argent pour des excursions ou d'autres dépenses scolaires leur soient acheminées. 
Une autre stratégie proposée par le collectif concerne la promotion de centres de services polyvalents à l'intention des enfants de la communauté. Parmi les exemples d'initiatives de ce genre, notons celle des organismes et de la communauté qui ont mis sur pied, en 1991, un programme de prévention intitulé Partir d'un bon pas pour un avenir meilleur. Ce programme s'adresse en priorité à tous les enfants âgés de quatre à huit ans dans deux quartiers à faible revenu de Sudbury, un à majorité francophone, l'autre à majorité anglophone. Parmi les caractéristiques de ces centres, on note l'absence de liste d'attentes, la gratuité des services et l'ouverture totale, c'est-à-dire que les gens n'ont pas besoin de raisons précises pour les fréquenter. Les membres défavorisés de ces communautés travaillent à la restauration de leur fierté culturelle. Comme Diallo et Reitsma-Street l'indiquent : «La culture a été utilisée... comme une force et un moyen pour le groupe francophone de se constituer et de s'affirmer.» (1995: 66).

Enfin, les membres du collectif ont décidé de s'attaquer aux préjugés et à la discrimination qui collent à la peau des familles et des quartiers à faible revenu. Par exemple, un certain nombre de personnes et de groupes se sont mobilisés pour supprimer une ligne téléphonique de délation contre les soit disant fraudeurs de l'aide sociale. Cette initiative s'est avérée efficace: «La ligne téléphonique de Sudbury a été supprimée en mars 1995 à la suite d'un débat public et d'une action concertée de ses opposants, dont des travailleurs sociaux travaillant pour des organismes locaux, des membres de l'Université Laurentienne et une coalition de représentants d'assistés sociaux et de divers organismes.» (ReitsmaStreet et Keck 1996: 41).

\section{Conclusion}

Les chiffres et les indices de l'impact de la pauvreté des enfants et des adolescents dans la région de Sudbury ne constituent pas un cas isolé. Dans une étude sur la pauvreté urbaine, Lockhead et 
Shillington (1996) ont trouvé qu'il y avait, dans des municipalités comme Hamilton, en 1990, un taux de pauvreté infantile de l'ordre de $26 \%$. Montréal-Nord connaît un taux de pauvreté incroyable de $42 \%$. Bien plus, la situation ne s'améliore ni à Sudbury ni ailleurs. Pour le Canada, le taux de pauvreté est en hausse continuelle, année après année : 16,9\% en 1990 pour les enfants âgés de 18 ans ou moins; 18,3 \% en 1991; 18,9\% en 1992 et $21,3 \%$ en 1993. Une faible diminution est enregistrée en 1994 avec un taux de 19,5\% (Ross et al. 1996). Contrairement à l'idée véhiculée par le premier Ministre du Canada, Jean Chrétien, voulant que le Canada n'ait rien à envier à quiconque quant à la qualité de vie offerte à ses citoyens, l'UNICEF rapporte que: «En 1996, le Canada était le deuxième pays parmi les dix-huit pays industrialisés au niveau de son taux de pauvreté chez les enfants» (cité dans le Sudbury Star: 12 juin 1996). Cette situation s'avère d'autant plus critique depuis les grandes coupures du gouvernement conservateur de l'Ontario, notamment celles d'octobre 1995 où les taux de prestation de l'aide sociale générale et des prestations familiales de l'Ontario ont été coupés de 21,6\%.

Il existe de nombreux renseignements sur les causes de la pauvreté, ainsi que sur les moyens d'y remédier. D'une part, la persistance de la pauvreté chez les enfants «reflète l'absence d'un consensus et d'un engagement national en ce qui concerne la responsabilité collective des Canadiens de contribuer aux soins à donner aux enfants canadiens et d'aider à soutenir la vie familiale» (Kitchen et al. 1991:55). D'autre part, cette persistance est attribuée à «l'action collective... [qui] est de plus en plus menacée et muselée par les décisions économiques des grandes entreprises multinationales ou transnationales, lesquelles ne sont pas imputables des répercussions de leurs décisions sur la vie quotidienne des familles et des enfants.» (Reitsma-Street et al.1994d: 153). Dans son ouvrage éloquent intitulé The Politics of Child Survival, Kent conclut: "Ceux qui gagneraient au changement n'ont pas le pouvoir de le provoquer, alors que ceux qui en ont le pouvoir n’y gagneraient rien.» (Kent 1991: 137). En dépit de la résolution visant à éliminer la pauvreté chez les enfants avant l'an 2000, adoptée par la Chambre des communes, et malgré toute 
l'information qui existe au sujet de la pauvreté et de ses effets sur les enfants en particulier, les gouvernements fédéral, provinciaux et municipaux refusent de s'engager à prendre des moyens réels pour résoudre le problème de la pauvreté. Nonobstant les coûts humains, la lutte contre la dette et contre le déficit sont devenus la priorité pour les gouvernements fédéral et provinciaux. Avec l'appui des corporations, les gouvernements ont réussi à convaincre la population canadienne qu'il faut accepter les coupures dans les programmes sociaux pour combattre le déficit. On a mis de côté le problème de la pauvreté chez les enfants mais, entre temps, le taux de la pauvreté continue de s'accroître.

L'ensemble des étapes et des stratégies décrites ci-haut sont possibles au niveau de toutes les municipalités. Nous pouvons faire en sorte que chacune d'elles accepte de discuter des enjeux et s'engage concrètement dans la lutte contre la pauvreté. Mais, cela n'est pas suffisant. Nous avons besoin de travailler, à une plus grande échelle, au développement de politiques gouvernementales visant à assurer une structure d'imposition progressiste, ou du moins plus équitable; le plein emploi; des services de garde à prix abordable pour assurer le bien-être de tous les enfants et adolescents du pays. Nous devons travailler à influencer les fonctionnaires responsables des politiques liées à la pauvreté; nous devons susciter des idées et des programmes créatifs, comme propose Jane Jenson (1993: 138) «d'autres espaces politiques pour accommoder la revendication démocratique.»

\section{Bibliographie}

BROWN, Jim, (1994). «Study will probe problem of Sudbury Students living and learning in poverty. Northern Life, $1^{\text {er }}$ juillet, B1.

CARRIÈRE, Richard et Adje VAN DE SANDE, (1994). «Children Under 18 on Social Assistance in the Region of Sudbury", dans Marge Reitsma-Street, éd. Child and Youth Poverty in Sudbury: A Follow-Up Report, School of Social Work, Laurentian University, Sudbury, 27-35.

CARRIÈRE, Richard, C. HEIN, Marge REITSMA-STREET, Adje VAN DE SANDE, (1992). La pauvreté chez les enfants et chez les jeunes dans la région de Sudbury, École de service social, Université Laurentienne. 
COMITÉ PERMANENT DE LA SANTÉ ET DU BIEN-ÊTRE SOCIAL, DES AFFAIRES SOCIALES, DU TROISIÈME ÂGE ET DE LA CONDITION FÉMININE, (1993). Promesse de l'an 2000: Éliminer la pauvreté des enfants, Imprimeur de la Reine pour le Canada, Approvisionnement et Services Canada, Ottawa.

CONSEIL NATIONAL DU BIEN-ÊTRE SOCIAL SOCIAL, (1992). Profil de la pauvreté, 1980 à 1990, Approvisionnements et Services Canada, Ottawa.

COMITÉ D'EXAMEN DE L'AIDE SOCIALE, (1988).Volume II, Transition, ministère des Services Sociaux et Communautaires de l'Ontario, Imprimeur de la Reine pour l'Ontario, Toronto.

DE LA RIVA, Paul, (1992). «Il y a 9420 enfants pauvres.» Le Voyageur, 27 mai, 5.

DIALLO, Lamine et Marge REITSMA-STREET, (1995). «Stratégies de survie et d'identité : les dynamiques culturelles dans un project d'intervention en prévention communautaire», Reflets: Revue ontaroise d'intervention sociale et communautaire, vol. 1, no. 1, 43-69.

GIROUARD, André, (1994). «Un taux de pauvreté plus élevé que la moyenne ontarienne», Le Voyageur, 30 novembre, 4.

HEIN, Carol, (1994). «Poverty Rates for Children,Youth and Families in the Sudbury Region», dans Marge Reitsma-Street (Ed.) Child and Youth Poverty in Sudbury: A Follow-Up Report, School of Social Work, Laurentian University, Sudbury, 8-26.

HEIN, Carol.,V. SMITH-DANYLIW, et A.M. KOOIMAN A.M., (1994). «The Effects of Poverty on Children: Perception of Social Assistance Recipients», dans Marge Reitsma-Street, éd. Child and Youth Poverty in Sudbury: A Follow-Up Report, School of Social Work, Laurentian University, Sudbury, 57-73.

JENSON, J. (1993). «De-constructing Dualities: Making Rights Claims in Political Institutions,» dans G. Drover et P. Kerans, éd. New Approaches to welfare theory, Aldershot, Hants: Gower House, 127-142.

KENT, George (1991). The Politics of Children's Survival (Praeger).

KITCHEN, Brigitte,Andrew MITCHELL, Peter CLUTTERBUCK and Marvyn NOVICK (1991). Unequal Futures:The Legacies of Child Poverty in Canada. The Child Poverty Action Group and the Social Planning Council of Metropolitan Toronto.

LAPP, Lorraine, (1996). "A Call for Action: Group Urges Local Leaders to Stem Tide of Job Losses», The Sudbury Star, 13 juin, A1.

LOCKHEAD, Clarence et Richard SHILLINGTON (1996). Un profil statistique de la pauvreté urbaine, Ottawa, Centre de statistiques internationales, Conseil canadien de développement social.

PENDER, Terry, (1992). «Thousands of Sudbury Children Live in Poverty, Study Concludes», The Sudbury Star, 21 mai, A1.

PENDER, Terry, (1994). «Child Poverty Living With The Stigma of Being Poor», The Sudbury Star, 24 novembre, B1.

PILON, Clarie, (1993). «Youth Poverty Does Exist», The Sudbury Star, 19 juin, A3.

PLOUFFE, Kim-Dominique, (1993). «Region Urged to Tackle Child Poverty With Policy», Northern Life, 21 novembre, A3.

PLOUFFE, Kim-Dominique (1994). «Canadians Must Take Care of Each Other-poverty Report», Northern Life, Novembre 25, A1.

REITMA-STREET, Marge et Jennifer KECK, (1996). «L'abolition, à Sudbury, d'une ligne délateur», Le Travailleur Social, vol. 64, no. 3, 41-54. 
REITSMA-STREET, Marge, (Ed.), (1994a). Child and Youth Poverty in Sudbury: A Follow-Up Report, School of Social Work, Laurentian University, Sudbury.

REITSMA-STREET, Marge, (1994b). «When There Is No Food Or Shelter: Food Bank and Crisis Housing», dans Child and Youth Poverty in Sudbury: A Follow-Up Report, School of Social Work, Laurentian University, Sudbury, 36-44.

REITSMA-STREET, Marge, (1994c). «Disturbing Trends, Promising Initiatives in the Struggle to Eliminate Child Poverty in Sudbury", dans Child and Youth Poverty in Sudbury: A Follow-Up Report, School of Social Work, Laurentian University, Sudbury, 74-83.

REITSMA-STREET, Marge, Richard CARRIÈRE,AdjeVAN DE SANDE, et Carol HEIN, (1994d). La pauvreté des enfants au Canada: trois perspectives. Le travailleur social, vol. 62, no. 4, 149-154.

ROSS, David P., Katherine SCOTT et Mark KELLY (1996). La pauvreté des enfants: quelles en sont les conséquences? Ottawa, Centre de statistiques internationales, Conseil canadien de développement social.

SAHAI,Vic, (1994). «Health and poverty in youth», dans Marge Reitsma-Street, éd.Child and Youth Poverty in Sudbury: A Follow-Up Report, School of Social Work, Laurentian University, Sudbury, 45-56.

SOCIAL PLANING COUNCIL (1994). Revealing Strengths Youth Conference, Sudbury.

ST. PIERRE, Denis (1992). «Region takes aim on child poverty», The Sudbury Star, $1^{\text {er }}$ juillet, B1.

SUDBURY BOARD OF EDUCATION (1995). Office of the Director. Guidelines for fees, fundraising and Field trips

THE SUDBURY STAR (1996). Canada gets low marks on child poverty, suicide, June 12: A1.

\section{Notes}

1. Les auteurs sont membres de l'École de service social de l'Université Laurentienne à Sudbury. Cet article n'aurait pas été possible sans l'appui de 29 organismes sociaux et communautaires de la région de Sudbury et sans l'apport de nos collègues Richard Carrière et Carol Kauppi. Les auteurs tiennent également à remercier la Fondation Laidlaw qui a financé les rapports de 1992 et 1994.

2. Ces municipalités sont Nickel Centre, Rayside-Balfour, Valley East, Walden, Onaping Fall et Capréol.

3. Notons ici certaines difficultés du langage. Le collectif en question est davantage une action concertée d'organismes et d'individus réunis dans un réseau d'intervention qu'un collectif formel. Pour les besoins du présent texte, nous parlerons d'un collectif. 